\title{
Schuldfähigkeit bei Personen, die wegen Nutzung von Missbrauchsabbildungen angeklagt sind
}

\author{
Johannes Fuß ${ }^{1}$ Alexander Voulgaris ${ }^{1}$ Peer Briken ${ }^{1}$ \\ Eingegangen: 1. Oktober 2020 / Angenommen: 2. Oktober 2020 / Online publiziert: 12. Oktober 2020 \\ (c) Der/die Autor(en) 2020
}

\section{Zusammenfassung}

Die Nutzung von Missbrauchsabbildungen (umgangssprachlich „Kinderpornografie“) und damit im Zusammenhang stehende Straftaten haben in den letzten Jahren an Bedeutung gewonnen. Der vorliegende Aufsatz beleuchtet die gängigen forensisch-psychiatrischen Kriterien zur Einschätzung der Schuldfähigkeit bei Personen, die wegen des Erwerbs, Besitzes oder der Verbreitung kinderpornografischer Schriften angeklagt sind. Liegt aus forensisch-psychiatrischer Perspektive eine schwere Sexualpathologie vor, die die psychiatrischen Voraussetzungen für eine sog. schwere andere seelische Abartigkeit erfüllt, so wird sich der Gutachter die Frage einer Steuerungsminderung stellen. Im Fall von Delikten im Zusammenhang mit der Nutzung von Missbrauchsabbildungen lassen sich die Kriterien aus den Mindestanforderungen jedoch aus Sicht der Autoren schlecht anwenden. Ein weiteres Problem bei der Begutachtung ist, dass sich die angeklagten Straftaten häufig über einen längeren Zeitraum verteilen und daher der Sachverständige ggf. nach überdauernden psychopathologischen Hinweisen einer geminderten Steuerungsfähigkeit suchen muss. Die sexuelle Dranghaftigkeit, die motivationale Steuerungsfähigkeit und die Desaktualisierungspotenz sind hilfreiche Konzepte, um die Steuerungsfähigkeit in diesem Zusammenhang einzuschätzen. Eine überdauernde forensisch-relevante Steuerungsminderung, die sich ggf. über mehrere Jahre hingezogen hat, wird im Zusammenhang mit der Nutzung von Missbrauchsabbildungen in der Regel nicht vorliegen und eher einen Sonderfall darstellen.

Schlüsselwörter Schuldfähigkeit $\cdot \S 21 \mathrm{StGB} \cdot$ Schuldminderung $\cdot$ Steuerungsfähigkeit $\cdot$ Begutachtung

\section{Criminal responsibility of persons accused of using child sexual abuse images}

\begin{abstract}
The use of child sexual abuse images (colloquially child pornography) and related crimes have gained in importance in recent years. The present paper examines the commonly used forensic psychiatric criteria for assessing the criminal responsibility of people accused of acquiring, possessing or distributing child sexual abuse images under German criminal law. If, from a forensic psychiatric perspective, there is a severe sexual pathology that fulfils the psychiatric criteria for the legal category of so-called severe other mental abnormalities, the forensic psychiatric expert will assess the question of diminished control over behavior. In the case of offences in connection with the use of child sexual abuse images, however, from the authors' perspective the established criteria for sexual offences are difficult to apply. A further problem in the assessment is that the accused crimes are often spread over a longer period of time and therefore the expert may have to search for persistent psychopathological indications of diminished control. Sexual urgency, motivational controllability and the power of deactualization are helpful concepts for assessing a loss or reduction of control in this context. An enduring forensically relevant reduction in control, which may have persisted for several years, will not usually be present in connection with the use of child sexual abuse images and will more likely represent a special case under German criminal law.
\end{abstract}

PD Dr. med. Johannes Fuß

jo.fuss@uke.de
Institut für Sexualforschung, Sexualmedizin und Forensische Psychiatrie, Universitätsklinikum Hamburg-Eppendorf, Martinistr. 52, 20246 Hamburg, Deutschland 
Keywords Legal responsibility $\S 21 \mathrm{German}$ criminal code $\cdot$ Culpability $\cdot$ Controllability $\cdot$ Expert testimony

Der Besitz von Missbrauchsabbildungen (umgangssprachlich „Kinderpornografie“) hat durch die Verbreitung digitaler Medien zugenommen (Franke und Graf 2016; Dekker et al. 2016), und so haben auch Straftaten im Zusammenhang mit der Nutzung von Missbrauchsabbildungen in den letzten Jahren an Bedeutung gewonnen. Die Aufdeckung schwerer Fälle von Kindesmissbrauch und von Handel mit Missbrauchsabbildungen hat in der Vergangenheit immer wieder zu politischen Forderungen nach härteren Strafen geführt. Dies war auch in den vergangenen Monaten, nachdem die Missbrauchsfälle von Münster bekannt wurden, erneut zu beobachten. Die forensische Psychiatrie hat sich in diesem Zusammenhang schon früh um eine Versachlichung der öffentlichen Diskussion um Missbrauchsabbildungsdelikte bemüht und mit wissenschaftlichen Daten versucht, sowohl die Täter als auch die Folgen für Opfer besser zu verstehen (z. B. Graf und Dittmann 2009).

Auf sachverständiger Ebene kann der forensische Psychiater Hinweise für eine relevante Einschränkung der Steuerungsfähigkeit feststellen, wenn die Täter schwer im Sinne einer schweren anderen seelischen Abartigkeit gemäß $\S \S 20,21$ StGB gestört sind. In den meisten Fällen, in denen der Erwerb, Besitz oder die Verbreitung kinderpornografischer Schriften ohne „Hands-on“-Delikte angeklagt ist, wird sich nicht die Frage einer Unterbringung im Maßregelvollzug gemäß $\S 63$, StGB stellen. Daher werden Sachverständige in der Regel lediglich beauftragt rechtlich zu prüfen, ob eine Minderung der Strafe aufgrund einer psychischen Störung in Betracht kommt. Prinzipiell hat die Rechtsprechung dargelegt, dass die Schuld aufgrund beeinträchtigter Steuerungsfähigkeit gemindert sein kann, ,wenn abweichende Sexualpraktiken zu einer eingeschliffenen Verhaltensschablone geworden sind, die sich durch abnehmende Befriedigung, zunehmende Frequenz, durch Ausbau des Raffinements und durch gedankliche Einengung auf diese Praktiken auszeichnen" (BGH, Beschluss vom 03.09.2015 - 1 StR 255/15, juris; BGH, Beschluss vom 06.07.2010, 4 StR 283/10 - juris; BGH vom 17.07.2007, 4 StR 242/07, NStZ-RR 2007, 337). Diese Formulierung, die noch auf die Konzepte sexueller Süchtigkeit von Giese (1962) zurückgeht, ist so weit gefasst, dass eine beeinträchtigte Steuerungsfähigkeit bei Erwerb, Besitz und Verbreitung kinderpornografischer Schriften zunächst nicht unplausibel wirkt. Tatsächlich lassen die Stichworte „,eingeschliffene Verhaltensschablone“, „,abnehmende Befriedigung“, „zunehmende Frequenz“ und „gedankliche Einengung" an typische Patienten denken, die sich hilfesuchend in Ambulanzen vorstellen, weil sie das Gefühl haben, ihren Konsum von Missbrauchsabbildungen nicht alleine in den Griff zu bekommen. Dennoch würde man bei der Mehrzahl dieser Patienten nicht prima facie von einer forensisch-relevanten Minderung der Steuerungsfähigkeit ausgehen. Die oben genannte Formulierung hilft dem psychiatrischen Sachverständigen an dieser Stelle also nur unzureichend weiter. Das Ziel des vorliegenden Artikels ist es, forensisch-psychiatrische Kriterien zu beleuchten, anhand derer die psychiatrischen Voraussetzungen für die Einschätzung der Schuldfähigkeit bei Patienten, die wegen des Erwerbs, Besitzes oder der Verbreitung kinderpornografischer Schriften angeklagt sind, beurteilt werden können.

Entsprechend dem zweistufigen Vorgehen gilt es zunächst, den Schweregrad der sexuellen Störung zu prüfen. Typische sexuelle Störungsbilder, die im Zusammenhang mit Erwerb, Besitz oder Verbreitung kinderpornografischer Schriften stehen, sind die pädophile Störung, sexueller Sadismus und die zwanghafte sexuelle Verhaltensstörung ${ }^{1}$, die sich in der ICD-11 unter den Impulskontrollstörungen finden wird, häufig in Kombination mit Persönlichkeitsstörungen oder -akzentuierungen. Mögliche Gründe, dass einzelne oder eine Kombination von mehreren dieser Störungen dem Eingangsmerkmal einer schweren anderen seelischen Abartigkeit gemäß der $\S \S 20,21$ StGB zugeordnet werden können, lassen sich mithilfe der folgenden Kriterien von Boetticher et al. (2007) prüfen:

- Die Sexualstruktur ist weitestgehend durch die paraphile Neigung bestimmt.

- Eine Ich-dystone (Ich-fremde) Verarbeitung führt zur Ausblendung der Paraphilie.

- Eine progrediente Zunahme und „Überflutung“ durch dranghafte paraphile Impulse mit ausbleibender Satisfaktion beherrschen zunehmend das Erleben und drängen zur Umsetzung auf der Verhaltensebene.

- Andere Formen soziosexueller Befriedigung stehen dem Beschuldigten aufgrund (zu beschreibender) Persönlichkeitsfaktoren und/oder (zu belegender) sexueller Funktionsstörungen erkennbar nicht zur Verfügung.

Obwohl dies nicht explizit in den Mindestanforderungen von Boetticher et al. benannt ist, leiten sich diese Kriterien vom Progredienzbegriff Schorschs ab, der die manchmal fast stereotypen Entwicklungen im Vorfeld von Sexualstraf-

\footnotetext{
${ }^{1}$ Im vorliegenden Artikel wird der Begriff ,zwanghafte sexuelle Verhaltensstörung" genutzt, um sich auf die Konzeptualisierung in der ICD-11 zu beziehen, wo außer Kontrolle geratenes Sexualverhalten als Impulskontrollstörung klassifiziert wird. Andere Konzepte z. B. der Hypersexualität oder sexuellen Süchtigkeit meinen ein vergleichbares klinisches Phänomen, werden aber aufgrund der genannten Konzeptualisierung in der ICD-11 voraussichtlich in Zukunft in der gutachterlichen Praxis eine geringere Rolle spielen.
} 
taten versucht hat, motivisch nachvollziehbarer zu machen, und dabei auch unbewusste psychodynamische Aspekte berücksichtigt haben wollte (Schorsch 1988). Ein ausgeprägtes Ausmaß der paraphilen Störung kann als eine Art prototypische Entwicklung angesehen werden, die zur Einschätzung führen kann, dass die psychiatrischen Voraussetzungen für eine sog. schwere andere seelische Abartigkeit gemäß der §§ 20, 21 StGB erfüllt sind. Die paraphile Störung würde typischerweise im Delikt einen (ggf. vorläufigen) progredienten „Höhepunkt“ erreicht haben. Hierbei sollte nicht übersehen werden, dass bei klassischen Hands-on-Sexualdelikten die paraphile Fantasiewelt auf die Verhaltensebene gegenüber einer realen Person gehoben wird, was bei dem Vorliegen einer pädophilen Störung und dem Tatvorwurf der Nutzung von Missbrauchsabbildungen im digitalen Raum nicht vergleichbar geschieht, ohne damit in Bezug auf die Nutzung von Missbrauchsabbildungen eine moralische Wertung verbinden zu wollen, denn selbstverständlich stehen hinter einer Vielzahl von Missbrauchsabbildungen reale Missbrauchshandlungen. Der fantasierte Übergriff gegen das Kind kann aber bei vielen Nutzern von Missbrauchsabbildungen (noch) verhindert werden und findet Ausdruck in der zielgerichteten Masturbation unter Verwendung illegalen Bildmaterials. Eine weitere Progredienz in den realen Raum hinein kann unterbunden werden, bleibt somit steuer- und kontrollierbar.

Nach Schorschs psychodynamischem Verständnis erklärt sich die Progredienz, die im Übrigen auch bei anderen Störungsbildern wie Zwängen oder Phobien auftreten könne, dadurch, dass die (sexuelle) Störung eine ,reparative, stützende Funktion zur Wiederherstellung bzw. zur Aufrechterhaltung des inneren Gleichgewichts" (Schorsch 1988, S. 348) habe. Sie habe also eine Funktion außerhalb des Sexuellen, indem sie z. B. Ängste lindere. Progredienz trete dann auf, wenn die übliche Inszenierung der sexuellen Störung (=das Symptom) das innere Gleichgewicht nicht mehr ausreichend stabilisiere. Die dann auftretende häufigere Wiederholung des Symptoms, die Giese den „süchtigen Verfall“ genannt hat (Giese 1962), beschreibt Schorsch als ein „verzweifeltes Sich-Klammern an das Symptom wie an einen Rettungsanker“ und „ein immer vergeblicheres Hoffen auf die reparative Funktion des Symptoms, das diese Funktion nicht mehr erfüllen kann" (Schorsch 1988, S. 348). So erkläre sich, dass die Befriedigung durch das Symptom abnehme und die Progredienz die Gefahr einer krisenhaften Zuspitzung innehabe. Verhaltenstherapeutisch würde man in diesem Zusammenhang von einer negativen Verstärkerfunktion des Symptoms sprechen, dessen Ausführung das Auftreten von negativen Gefühlen verhindert (Briken 2020). Der Verlust der „reparativen Funktion“ könnte z.B. durch Habituation oder Extinktion erklärt werden, je nachdem, ob man davon ausgeht, dass die Assoziation zwischen Symptom und reparativer Funk- tion angeboren oder erlernt wurde. Im Zusammenhang mit Missbrauchsabbildungsdelikten müsste eine entsprechende Progredienz sich also nicht nur durch immer extremere Darstellungen und häufigere Nutzung zeigen, sondern v.a. durch Verlust der Satisfaktion und der stabilisierenden Funktion. Ersteres wird sich unschwer auf Verhaltensebene nachweisen lassen, wenn ein Proband z. B. zunächst legales Bildmaterial zur Masturbation genutzt hat, dann computergenerierte Abbildungen oder „Posing“-Bilder (also die Darstellung von Kindern in sexuellen Posen) und schließlich Darstellungen sexuellen Missbrauchs mit Penetration und Gewalt (Taylor et al. 2001). Aus klinischer Erfahrung ist diese Art der Progredienz aber geradezu typisch für Probanden, die sich wegen Missbrauchsabbildungsdelikten in Präventionsambulanzen vorstellen oder angezeigt werden. Sie ist viel stärker ein kriminologisches Merkmal und u.U. prognostisch bedeutsamer als für die Einschätzung der Schuldfähigkeit. Damit die sexuelle Störung einer sog. schweren anderen seelischen Abartigkeit gemäß der $\S \S 20,21$ StGB zugeordnet werden könnte, sollten die Betroffenen in der Regel zusätzlich Anzeichen zeigen, dass die stabilisierende Funktion durch das Symptom schrittweise abhandengekommen ist. Dies kann sich auf der Verhaltensebene im Alltag durch deutliche Probleme in der Beziehungs- und Bindungsfähigkeit zeigen, sodass den Probanden keine anderen Möglichkeiten mehr zur sexuellen Befriedigung vorliegen. Die Sexualanamnese wird im Regelfall frühe und deutliche Auffälligkeiten zeigen, und es wird keine oder gescheiterte Versuche von partnerschaftlichem Geschlechtsverkehr gegeben haben. Häufig wird eine komorbide Persönlichkeitsstörung oder zumindest -akzentuierung vorliegen, die es der Person erschwert haben mag, trotz der Sexualpathologie sexuelle Befriedigung zu erlangen. An dieser Stelle wäre also nicht von einem gut integrierten Menschen auszugehen, der Beruf und Familie hat und im sozialen Handeln weitestgehend unauffällig ist. Vielmehr ist zumindest im Längsschnitt meist von einer deutlichen Herabsetzung der allgemeinen psychosozialen Leistungsfähigkeit auszugehen. In der forensischen Praxis werden zur Beurteilung des Schweregrades der störungsbedingten Einbußen häufig Vergleiche mit den Defiziten im Rahmen einer psychotischen Erkrankung, und somit einer forensisch relevanten krankhaften seelischen Störung, herangezogen (siehe auch psychopathologisches Referenzsystem nach Saß [1991] und strukturell-sozialer Krankheitsbegriff nach Rasch (Konrad [2018]). Liegt aus forensisch-psychiatrischer Perspektive eine schwere Sexualpathologie vor, die die psychiatrischen Voraussetzungen für eine sog. schwere andere seelische Abartigkeit erfüllt, so wird sich der Gutachter anschließend die Frage einer Steuerungsminderung stellen. Aufgehobene Einsichtsund Steuerungsfähigkeit wird man in diesen Fällen kaum annehmen. 


\section{Die Steuerungsfähigkeit}

Besteht ein symptomatischer Zusammenhang zwischen vorgeworfenem Delikt und der sexualpathologischen Progredienz, lassen sich die folgenden Kriterien aus den Mindestanforderungen für Schuldfähigkeitsgutachten heranziehen, die für eine Minderung der Steuerungsfähigkeit bei Sexualstraftaten sprechen können (Boetticher et al. 2007):

- konflikthafte Zuspitzung und emotionale Labilisierung in der Zeit vor dem Delikt mit vorbestehender und länger anhaltender triebdynamischer Ausweglosigkeit,

- Tatdurchführung auch in sozial stark kontrollierter Situation,

- abrupter, impulshafter Tatablauf, wobei jedoch ein paraphil gestaltetes und zuvor (etwa in der Fantasie) „durchgespieltes" Szenario kein unbedingtes Ausschlusskriterium für eine Verminderung der Steuerungsfähigkeit ist, sofern dieses Szenario der (den) diagnostizierten Paraphilie(n) entspricht und eine zunehmende Progredienz nachweisbar ist,

- archaisch-destruktiver Ablauf mit ritualisiert wirkendem Tatablauf und Hinweisen für die Ausblendung von AuBenreizen,

- konstellative Faktoren (z.B. Alkoholintoxikation, Persönlichkeitsstörung, eingeschränkte Intelligenz), die u. U. auch kumulativ eine erheblich verminderte Steuerungsfähigkeit bedingen können.

Wenn man diesen Kriterienkatalog heranzieht, um die Steuerungsfähigkeit bei Missbrauchsabbildungsdelikten einzuschätzen, fällt zunächst auf, dass sich die Kriterien aus den Mindestanforderungen schwer anwenden lassen. Wenn der Erwerb, Besitz und die Verbreitung von kinderpornografischen Schriften als zeitlich begrenztes Ereignis angeklagt sind, mag es noch möglich sein, eine emotionale Labilisierung im Vorfeld und den Erwerb in sozial stark kontrollierten Situationen festzustellen. Was jedoch unter einem archaisch-destruktiven oder abruptimpulshaften Erwerb von kinderpornografischen Schriften $\mathrm{zu}$ verstehen ist, stellt sich bereits weniger verständlich dar. An dieser Stelle scheinen die gängigen Kriterien also schlecht anwendbar. Noch komplizierter wird es, wenn der Proband wegen Nutzung von Missbrauchsabbildungen angeklagt ist, die sich ggf. über mehrere Jahre hingezogen hat. Selbst wenn für einzelne Phasen entsprechend den oben stehenden Ausführungen von einer forensisch relevanten Minderung der Steuerung auszugehen wäre, so lässt sich dies nicht einfach auf den gesamten Zeitraum des Dauerdeliktes ausdehnen.

Beide Probleme sollen im Folgenden diskutiert werden. Zunächst wollen wir forensisch-psychiatrische Konzepte vorstellen, die nach unserer Einschätzung besser für die
Beurteilung der Steuerungsfähigkeit bei Missbrauchsabbildungsdelikten geeignet sind. Anschließend widmen wir uns dem Problem der über einen längeren Zeitraum hingezogenen Straftaten.

\section{Die sexuelle Dranghaftigkeit}

Vor einiger Zeit wurde sexuelle Dranghaftigkeit als Kernmerkmal von Briken und Müller (2014) zur Einschätzung der Steuerungsfähigkeit bei Sexualstraftaten vorgeschlagen. Diesem Vorschlag folgte eine Überarbeitung initial vorgeschlagener Kriterien durch Brunner et al. (2016). Neben der sexuellen Dranghaftigkeit sollen zwei weitere Items, die sich ebenfalls im etablierten Instrument ACUTE-2007 befinden (Matthes und Rettenberger 2008), welches eigentlich für die Prognose bei Sexualdelinquenz genutzt wird, für die Einschätzung der Steuerungsfähigkeit hilfreich sein:

- Dranghaftigkeit: nicht in der Lage, paraphile Impulse zu kontrollieren, auch ohne situative Außenreize paraphil okkupiert, Sex als Coping-Strategie, Auftreten in sozial stark kontrollierten Situationen,

- emotionaler Zusammenbruch: Anzeichen eines emotionalen Zusammenbruchs, Hilfsbedürftigkeit, subjektives Krankheitserleben, Verlust von Coping-Strategien,

- Zusammenbruch sozialer Unterstützung: im Tatzeitraum bedeutsamer Verlust positiver sozialer Unterstützung und/oder partnerschaftlicher Beziehungen.

Notwendig sei laut Briken und Müller für die Einschätzung einer forensisch relevanten Minderung der Steuerungsfähigkeit eine sexuelle Dranghaftigkeit. Dagegen spräche die Möglichkeit, in vergleichbaren Situationen im Tatzeitraum anders zu handeln, gegen eine relevante Minderung der Steuerungsfähigkeit. Trotz der Ähnlichkeit der Beschreibung ist Dranghaftigkeit nicht mit dem Vorliegen einer zwanghaften sexuellen Verhaltensstörung gleichzusetzen, sodass hier kein Automatismus zwanghafte sexuelle Verhaltensstörung $=$ verminderte Steuerungsfähigkeit angenommen werden sollte. Vielmehr zeichne sich laut Briken und Müller das Merkmal durch „das Verhältnis zwischen sexueller Dranghaftigkeit und sexueller Inhibition, also der Fähigkeit entgegen sexueller Wünsche, Motive und Emotionen zu handeln, sodass sexuelle Impulse nicht unmittelbar in Handlung umgesetzt werden", aus. Auch eine zeitlich lang anhaltende und letztlich überfordernde Überkontrolle könne auf Krankheitswertigkeit hinweisen. Flexible sexuelle Selbstkontrolle spreche eher gegen eine schwere Störung. Es seien für die Einschätzung sowohl subjektive als auch beobachtbare Merkmale (z.B. unterschiedliche Reaktionen in vergleichbaren sexuellen Situationen) heranzuziehen (Briken und Müller 2014). Zu kurz gegriffen wäre es zu meinen, dass die nichtunumstrittene Zuordnung der zwanghaften sexuellen Verhaltensstörung in der zukünf- 
tigen ICD-11 in die Gruppe der Impulskontrollstörungen (Fuss et al. 2019) darauf hinweist, dass hier automatisch eine forensisch relevante Einschränkung der Selbstkontrollfähigkeiten vorliegt. Wie bei den paraphilen Störungen muss auch hier die Schwere der Störung sorgfältig geprüft werden und dürfte eher nur in Ausnahmefällen die Kriterien einer schweren Störung erfüllen. Ebenso wenig kann ohne Weiteres auf eine forensisch relevante Steuerungsminderung geschlossen werden. Dieses wäre sonst auch bei allen anderen Impulskontrollstörungen anzunehmen.

Die Kriterien emotionaler Zusammenbruch und Zusammenbruch der sozialen Unterstützung untermauerten die Einschätzung, seien aber nach den Kriterien von Briken und Müller nicht zwingend notwendig. Weitere Faktoren (wie z.B. akute Alkoholisierung) sollten, entsprechend den Mindestanforderungen als konstellative Faktoren berücksichtigt werden (Boetticher et al. 2007). Ähnlich wie bei den Kriterien der Mindestanforderungen beziehen sich zumindest der emotionale Zusammenbruch und der Zusammenbruch sozialer Unterstützung auf ein zeitlich begrenztes Ereignis und eben nicht auf einen mehrere Jahre andauernden Zustand, sodass diese für die Bewertung eines sich länger hinziehenden Besitzes von kinderpornografischen Schriften im Sinne eines Dauerdelikts nur eingeschränkt anwendbar erscheinen.

Die Kriterien von Briken und Müller wurden nach einer Überarbeitung bereits hinsichtlich ihrer Interrater-Reliabilität mit den oben genannten Kriterien der Mindestanforderungen verglichen und zeigten höhere Reliabilitätswerte. Dies könnte in der ausführlicheren Operationalisierung der Kriterien begründet sein. Allerdings wurde auch anhand dieser Kriterien lediglich eine ungenügende bzw. moderate Übereinstimmung (je nach statistischer Methode) bezogen auf das Vorliegen einer erheblich verminderten Steuerungsfähigkeit festgestellt (Dobbrunz et al. 2020). Hier besteht also sicher weiterer Forschungsbedarf und auch hinsichtlich der Nutzung von Missbrauchsabbildungen noch keine eindeutig geklärte Vorgehensweise.

\section{Motivationale Steuerung}

Eine Betrachtung der „motivationalen“ und „exekutiven“ Steuerung, wie von Kröber vorgeschlagen, kann ebenfalls hilfreich sein, um die Steuerungsfähigkeit bei Missbrauchsabbildungsdelikten einzuschätzen (Kröber 2016). Letztere fokussiert dabei auf den Ablauf des Tatgeschehens - wirkt dieser überlegt, situationsadäquat, kontrolliert und umsichtig, so spricht dies gegen eine relevante Störung der exekutiven Steuerung. Am Beispiel typischer Missbrauchsabbildungsdelikte würde damit in der Regel vieles gegen eine Störung der exekutiven Steuerung sprechen, weil die Personen für gewöhnlich kontrolliert vorgehen und manchmal sogar Schutzmaßnahmen durch Nutzung eines sicheren Browsers oder Versuche, die eigene IP-Adresse zu verschlüsseln, zeigen oder das Verhalten sich auf Situationen beschränkt, in denen sich die Person vermeintlich sicher fühlt. Eine Störung der exekutiven Steuerung, die eher bei hirnorganischen Störungen oder Intoxikationen zu erwarten wäre, liegt daher in der Regel bei Missbrauchsabbildungsdelikten nicht vor. Kröber beschreibt demgegenüber die motivationale Steuerungsfähigkeit als „die Fähigkeit, das eigene Handeln auch bei starken Wünschen und Bedürfnissen normgerecht zu kontrollieren und die Ausführung normwidriger Motivationen zu inhibieren“ (Kröber 2016, S. 186). Es geht also erneut um die Fähigkeit, trotz intensiver sexueller Motive oder Wünsche auf der Verhaltensebene Handlungen zu inhibieren. Ein Verlust der motivationalen Steuerung wäre beim Konzept der sexuellen Dranghaftigkeit zu prüfen, sodass sich beide Konzepte in der gutachterlichen Praxis ergänzen können. Als Drittes kann die Steuerung durch die Desaktualisierungspotenz entsprechend dem Konzept von Janzarik (1993) betrachtet werden.

\section{Desaktualisierung}

Der Begriff der Desaktualisierung geht auf den Strukturbegriff der Heidelberger Schule unter Janzarik zurück (Janzarik 1988). Desaktualisierung meint die Fähigkeit, „Triebbedürfnisse, Impulse und motorische Reaktionen“ zu unterdrücken, ,aufzuschieben, nachzutragen, Umwege in Kauf zu nehmen" oder darauf zu verzichten. Das strukturelle Gefüge der Psyche werde durch die Aktualisierungsund Desaktualisierungsbereitschaft gekennzeichnet. Am Beispiel von Missbrauchsabbildungsdelikten würde es also um die Fähigkeit gehen, sexuelles Begehren nach Missbrauchsabbildungen beiseitezuschieben, wenn dieses als unerwünscht oder störend empfunden wird. Wenn das Desaktualisierungsvermögen verloren gegangen ist, ließen sich sexuelle Impulse nicht mehr entschärfen, sie zwängen zur Umsetzung auch in sozial kontrollierten Situationen und überfluteten die Betroffenen, ohne dass es einen Anstoß durch Außenreize benötige. Anders als die Dranghaftigkeit bei Müller und Briken und die motivationale Steuerung von Kröber, bei denen es um die Umsetzung auf Handlungsebene geht, meint Desaktualisierung auch die Fähigkeit, Wahrnehmungen, Affekte und Vorstellungen zu unterdrücken, also Verhaltenskontrolle auf innerpsychische Abläufe auszuüben und diese zu entschärfen oder zu verdrängen. Damit ist das Modell von Janzarik vielleicht am sensibelsten, um frühe Anzeichen von Progredienz, die sich noch nicht auf der Handlungsebene manifestieren, festzustellen. Die psychopathologische Bedeutung dieser frühen Anzeichen, lässt sich anhand von Schorschs Schilderungen der affektiven Ausgangssituation vor Sexualdelikten verstehen. Auch Schorsch bezog sich primär auf einen innerpsychi- 
schen Kontrollverlust, der sich evtl. erst spät im sozialen Handeln niederschlägt. Schorsch beschrieb eine immer stärkere Okkupierung mit sexuellen Fantasien, eine Einengung, Isolierung, eine zunehmende innere Beunruhigung und Bedrängnis, immer vehementere Impulse, die Fantasie in die Realität umzusetzen, oft verbunden mit starken Ängsten vor dem Impulsdurchbruch und vor Kontrollverlust (Schorsch 1988, S. 349). Diese psychopathologischen Symptome, die sich zunächst nicht auf der Verhaltensebene niederschlagen müssen, können in der gutachterlichen Praxis mit dem Verlust der Desaktualisierungspotenz beschrieben werden, ohne dass ein Sachverständiger zwangsläufig mit dem Strukturbegriff der Heidelberger Schule arbeiten muss. Allerdings sind sie auch nur aus der Anamnese und kaum über Verhaltensbeobachtung zu klären und damit anfällig für Täuschung, Antworten im Sinne sozialer Erwünschtheit und Fehlinterpretationen durch Untersuchende.

Zusammenfassend kann eine Betrachtung dieser drei Konzepte bei der Einschätzung der Steuerungsfähigkeit bei Missbrauchsabbildungsdelikten hilfreicher als die Kriterien aus den Mindestanforderungen sein. Da die Kriterien von Briken und Müller aktuell der wissenschaftlichen Untersuchung unterzogen werden, wäre es wünschenswert, wenn die Überflutung, Okkupierung und Einengung auf psychischer Ebene aufgrund von Verlust der Desaktualisierungspotenz in der Operationalisierung der sexuellen Dranghaftigkeit Berücksichtigung fänden. Wünschenswert wäre auch, wenn dabei eine Terminologie gewählt würde, die nicht zwangsläufig auf den Heidelberger Strukturbegriff Bezug nimmt, sodass Gutachter aus verschiedenen Denkschulen und Therapierichtungen in Zukunft damit arbeiten können.

Im Folgenden soll das zweite Problem diskutiert werden, nämlich wie Schuldfähigkeit beurteilt werden kann, wenn die Frage des Gerichts nach einer Minderung der Steuerungsfähigkeit sich auf einen langen Zeitraum, der sich über Jahre erstrecken kann, bezieht.

\section{Der Zeitraum der Delikte}

Bei Erwerb, Besitz und Verbreitung kinderpornografischer Schriften handelt es sich häufig nicht um ein akutes Ereignis, sondern um Verhalten, das sich über einen längeren Zeitraum hingezogen hat. Daher müsste der Sachverständige nach überdauernden psychopathologischen Hinweisen einer gestörten Desaktualisierung/motivationalen Steuerung/sexuellen Dranghaftigkeit suchen.

Folgt man Schorschs psychodynamischem Verständnis von Sexualstraftaten, so könnte der Nutzung von Missbrauchsabbildungen bei diesen Probanden langfristig eine „reparative, stützende Funktion zur Wiederherstellung bzw. zur Aufrechterhaltung eines inneren Gleichgewichts“ zu- kommen - also eine Funktion außerhalb des Sexuellen. Die Nutzung von Missbrauchsabbildungen könnte einerseits das Resultat einer progredienten Entwicklung sein, andererseits dazu führen, dass diese zu einem Ende kommt und sich eine Phase der Stabilisierung einstellt. Dies entspricht einer Form des klinischen Eindrucks. Bei sich über mehrere Jahre hinziehenden Delikten ist typischerweise ein Auf und $\mathrm{Ab}$ zu beobachten, in dem in Zeiten zunehmender Bedrängnis (z.B. durch beruflichen Stress, zwischenmenschliche Konflikte oder Wegfall von Coping-Mechanismen) die Nutzung zunimmt, während sie dann stabil verlaufen oder ggf. wieder abnehmen kann, wenn sich die äußeren Bedingungen verbessern. Durch den strafbaren Besitz könnte der Proband also paradoxerweise Handlungsspielräume zurückgewinnen, die es ermöglichen, die normwidrigen Handlungen also in diesem Falle das Suchen nach und Beschaffen von Missbrauchsabbildungen abzubauen. Konkret könnte die Person nicht mehr weitersuchen, die bereits bestehenden Dateien löschen, sich in einer klinischen Einrichtung vorstellen, eine Psychotherapie beginnen oder den Internetanschluss kündigen. Selbst bei schwer gestörten Probanden (im Sinne einer sog. schweren anderen seelischen $A b$ artigkeit) kann nach einer Stabilisierung und bei fehlender Progredienz wieder ein verbessertes Hemmungsvermögen vorliegen. Dies gilt insbesondere, wenn sich Zeiten nachweisen lassen, in denen die Probanden nicht von sexuellen Symptomen überflutet waren. Typischerweise können Hinweise dafür eine fortgeführte berufliche Betätigung oder die Wiederaufnahme sozialer Beziehungen sein, bei der die Probanden sich nicht den dranghaften sexuellen Symptomen ausgesetzt erleben. Nur wenn überdauernd eine Okkupierung und Einengung auf psychischer Ebene festzustellen sind (also in den verschiedenen sozialen Situationen, in denen sich der Proband bewegt), kann auch eine überdauernde forensisch-relevante Steuerungsminderung, die sich ggf. über mehrere Jahre hingezogen hat, diskutiert werden. Bei der Formulierung wird klar: Hier wird es sich eher um einen Sonderfall handeln. Von gutachterlicher Seite kann es hilfreich sein, die Einschätzung der Steuerungsfähigkeit sowohl für den Erwerb, Besitz und die Verbreitung kinderpornografischer Schriften als auch für unterschiedliche Zeiträume gesondert durchzuführen, wenn kein Dauerdelikt sondern juristisch Tatmehrheit angenommen wird.

In vielen Fällen dürfte aber nach ,Überschreitung des Rubikon“, also nachdem bereits Missbrauchsabbildungen erworben und besessen wurden, ein Prozess der Selbstkorrumpierung (Janzarik 1993; Kröber 1993) einsetzen, der dazu führt, dass die Nutzung von Missbrauchsabbildungen fortgeführt wird. Diese Form der Fortführung wäre also nicht Ausdruck einer Steuerungsminderung, sondern Ausdruck einer Selbstkorrumpierung oder auch kognitiven Verzerrung im Sprachgebrauch der kognitiven Verhaltenstherapie bzw. Verleugnung und Rationalisierung in der 
Sprache psychodynamischer Abwehrmechanismen. Darunter versteht sich das Sammeln von Argumenten, die für die weitere Nutzung der Missbrauchsabbildungen sprechen oder deren Nutzung mit Argumenten wie ,die Darstellungen wurden ja sowieso schon hergestellt“ oder ,ich habe dafür ja nichts bezahlt und habe daher keinen Markt unterstützt" legitimieren. Am Beispiel von Straftaten in Bezug auf Missbrauchsabbildungen sind in vielen Fällen die Worte Kröbers zutreffend, dass die ,Entscheidung, nicht anders zu wollen, tage- und wochenlang immer aufs Neue getroffen“ wurde (Kröber 2007, S. 188).

Zusammenfassend ist bei sich über einen längeren Zeitraum hinziehenden Delikten im Zusammenhang mit Missbrauchsabbildungen meist nicht davon auszugehen, dass sich die Person fortlaufend in einem Zustand befunden hat, in dem die Steuerungsfähigkeit forensisch-relevant gemindert war. Insgesamt sollte sich die forensische Psychiatrie in diesem Zusammenhang nicht instrumentalisieren lassen, gesamtgesellschaftliche Entwicklungen hin zu Strafverschärfung bei Missbrauchsabbildungsdelikten ,,auszuhebeln“, weil die Strafen ggf. zu drakonisch wirken. Unten stehende Fallvignetten sollen anhand von Fällen die vorgetragenen Argumente verdeutlichen.

\section{Fallvignette 1}

Der 40-jährige Herr A. ist wegen des Besitzes kinderpornografischer Schriften angeklagt. Er schildert, dass er um die Pubertät erstmals Fantasien gehabt habe, in denen er sexuell mit Kindern verkehrt und diese gefesselt und geschlagen habe. Über die Jahre habe er diese Fantasien immer weiter ausgebaut, habe sich Szenarien überlegt, wie er Kinder als „Sexsklaven“ halten könne und dazu masturbiert. Andere sexuelle Fantasien hätten ihn kaum erregt, und er habe bis ins frühe Erwachsenenalter keine zwischenmenschlichen sexuellen Erfahrungen gemacht. Nachdem er seinen Beruf aufgenommen habe, hätten mehrere Male Frauen ein Interesse an ihm gezeigt. Er habe sich mit diesen auf deren Initiative getroffen und auch einen Beziehungswunsch gehabt, allerdings habe er sich nicht vorstellen können, mit ihnen sexuell zu verkehren und daher die Beziehungsversuche abgebrochen. Zweimal sei es dabei zum Versuch von Geschlechtsverkehr gekommen. Die Versuche seien aber gescheitert, da er nicht erregt gewesen sei. Unter seinen sexuellen Fantasien habe er zeitlebens gelitten und häufig nach der Selbstbefriedigung Ängste gehabt, dass er sich eines Tages so sehr in seine Fantasien ,hineinsteigern“ würde, dass er eine entsprechende Handlung ausführe. Im Alltag habe er den Kontakt zu Kindern eher vermieden, da er die spontan auftretende sexuelle Erregung beim Kontakt mit diesen unangenehm erlebt und Schuldgefühle gehabt habe. Um das Jahr 2000 habe er erstmals im Internet nach Bild- material gesucht, auf dem Kinder sexuell missbraucht werden. Er sei in dieser Zeit nach einem weiteren gescheiterten Beziehungsversuch enttäuscht gewesen und habe beruflich unter Druck gestanden. Er wisse noch, dass er dabei sehr stark sexuell erregt gewesen sei. Im Nachhinein habe er das Bildmaterial gelöscht und Scham empfunden. Es sei in der Folge aber immer wieder dazu gekommen, dass er entsprechende Dateien gesucht habe, und irgendwann habe er diese auch nicht mehr gelöscht, sodass er sich eine Sammlung an Missbrauchsabbildungen zugelegt habe. Er habe gewusst, dass dies illegal ist, aber sich eingeredet, dass er niemandem schade, da er ja nicht dafür bezahlt habe. Seine Sexualität habe er in den folgenden Jahren als immer dranghafter erlebt. Fast jeden Abend habe er für mehrere Stunden nach entsprechenden Abbildungen gesucht und sich dabei selbstbefriedigt. Manchmal habe dieses Verhalten bis in den Morgen angehalten, aber er habe es beenden können, wenn berufliche Verpflichtungen angestanden hätten. Zu weiteren Beziehungsversuchen sei es in den letzten Jahren nicht mehr gekommen. Irgendwann habe er das Gefühl gehabt, dass er keine Kontrolle mehr über sein Handeln habe und der Konsum für ihn alternativlos sei, da er große Teil seiner freien Abende so verbracht habe und sozial zurückgezogen gelebt habe. Gelegentlich habe er noch seine Eltern und Freunde getroffen und an diesen Tagen keine Missbrauchsabbildungen angeschaut. Seinen beruflichen Verpflichtungen sei er nachgekommen, da er an sein Verhalten hohe Ansprüche stelle und es ihm wichtig sei, dass man sich auf ihn verlassen könne. Spontan auftretende sexuelle Fantasien während der Arbeit erlebe er als störend und lenke sich deshalb ab. Das Suchen und Herunterladen von Missbrauchsabbildungen sei in den vergangenen Jahren sein einziger Weg gewesen, seine Sexualität auszuleben. Phasenweise sei dies verstärkt aufgetreten, wenn er Konflikte oder beruflichen Stress gehabt habe, zu anderen Zeiten habe er es auf ein zeitlich geringeres Maß reduzieren können. Die Nutzung von Missbrauchsabbildungen habe nur abends stattgefunden, wenn er allein zu Hause gewesen sei und sich habe entspannen können und sicher gefühlt habe. Er habe seine Fantasien nie in die Tat umgesetzt und wolle dies unter keinen Umständen. Er leide weiterhin unter seinen sexuellen Fantasien.

\section{Fazit}

In diesem Fall liegt eine schwere Sexualpathologie vor, die das Vorliegen einer sog. schweren anderen seelischen Abartigkeit aufgrund der Kombination von pädophilen, sadistischen und hypersexuellen Symptomen wahrscheinlich macht. Die Kriterien 1, 2 und 4 von Boetticher et al. (2007) können im vorliegenden Fall für das Vorliegen einer sog. schweren anderen seelischen Abartigkeit sprechen. Außer- 
dem ist eine gewisse Progredienz langfristig, aber nicht in den zurückliegenden Monaten erkennbar.

Dennoch dürften die sexuellen Impulse trotz der subjektiven Angabe eines Kontrollverlustes für den Probanden hemmbar sein, wenn dies (z.B. im familiären und beruflichen Kontext oder bei anderweitigen Verpflichtungen) notwendig ist. Konkret wirkt die motivationale Steuerung vorhanden: Die exzessive nächtliche Beschäftigung mit Missbrauchsabbildungen kann beendet werden, wenn berufliche Verpflichtungen dies verlangen; dranghafte sexuelle Fantasien werden beiseitegeschoben, wenn diese als unerwünscht erlebt werden. Damit ist es dem Probanden möglich, sowohl auf Verhaltens- als auch psychischer Ebene seine sexuellen Wünsche zu desaktualisieren. Die Symptomatik tritt ausschließlich auf, wenn der Proband sich diese ,zubilligt“, weil er abends seine Verpflichtungen erledigt hat und sich sicher fühlt. Obwohl er seine Sexualität als Coping-Strategie einsetzt und sie als zunehmend dranghaft erlebt, ist er ohne situative Außenreize nicht paraphil okkupiert. Ihm stehen alternative Handlungsstrategien zur Verfügung, die er aber nicht anwendet. Das Ausbleiben der Löschung von Missbrauchsabbildungen hat sich mit der Zeit eingeschlichen. Klare psychopathologische Gründe, die sich auf die sexuelle Störung zurückführen lassen, scheinen dafür weniger ursächlich.

\section{Fallvignette 2}

Der Mitte 20-jährige Herr B. ist wegen Besitz und Erwerb von kinderpornografischen Schriften angeklagt. Seit der frühen Grundschulzeit habe er für ausgesuchte Mitschüler geschwärmt, und, während er älter wurde, seien die ersehnten Objekte seiner Schwärmereien unverändert jung geblieben. Dabei habe es im Verlauf vereinzelt Versuche gegeben, Kontakt zu jüngeren Mitschülern aufzunehmen, die jedoch wiederholt gescheitert seien, womit aufkommende Beziehungswünsche in der Fantasie verhaftet blieben. Mit dem Fortschreiten der Pubertät habe er begonnen, seine sexuellen Wünsche im Internet zu befriedigen. Er habe gezielt nach sexuellen Missbrauchsabbildungen gesucht, wobei ihn der abgebildete ausgeübte Oralverkehr bei präpubertären Jungen am meisten und bis zum Höhepunkt erregt habe. Nach dem erfolgreichen Schulabschluss sei Herr B. zur Aufnahme eines Studiums verzogen und habe fortan in einer eigenen Wohnung gelebt. Diese habe er nach seinen Vorlieben eingerichtet, so habe er die Wände mit Postern von Kinderstars behangen, habe entsprechendes Kinderspielzeug besessen und auch medial verschiedene Kinderformate im Fernsehprogramm verfolgt. Im Verlauf habe die Sehnsucht nach einer Beziehung zu einem Kind zugenommen, dazu auch die Selbstbefriedigung durch den Gebrauch von Missbrauchsabbildungen. Er betont in der
Untersuchung, dass er damit keinen eigenen Markt unterstütze, und die Kinder durch diese Erfahrungen ,eine schöne Zeit erleben“"würden. Den Kontakt zur Familie habe er nach und nach abgebrochen, Freundschaften habe er nicht aufbauen können aufgrund der Angst davor, als „Pädophiler erkannt“" zu werden. Das Erststudium habe er aufgrund von schlechten Leistungen abbrechen müssen, und auch das seiner Meinung nach „leichtere“ zweite Studium würde sich hinziehen und sei aktuell gefährdet, da er wiederholt Lehreinheiten aufgrund von durchgemachten Nächten vor dem Rechner und der zunehmend intensiven Beschäftigung mit den Missbrauchsabbildungen verpassen würde. Bei seinem Nebenjob sei er im Verlauf als unzuverlässiger Mitarbeiter aufgefallen, er sei häufig zu spät oder auch gar nicht erschienen, da er nachts regelmäßig mehrstündig im Internet nach „,den perfekten“ Missbrauchsabbildungen gesucht habe. Die Kollegen hätten ihn als antriebslos, demotiviert und müde erlebt. Ebenso sei er auf der Arbeit vereinzelt verwarnt worden, da er auffällig viele Pausen auf der Toilette machen würde, wobei er dort regelmäßig mithilfe seines Telefons unter Nutzung von Missbrauchsabbildungen masturbiert habe. Dies sei v. a. vorgefallen, wenn er besonders attraktive Jungen gesehen habe. Dann sei die sexuelle Lust als überwältigend erlebt worden, und er habe seinen Trieb schnellstmöglich „erledigen“ müssen, wobei er wiederholt die Missbrauchsabbildungen aufgerufen habe. Deshalb habe er ausgewählte Missbrauchsabbildungen immer auf seinem Mobiltelefon bei sich getragen. Er könne in diesen Momenten an nichts anderes denken, könne die aufkommenden sexualisierten Gedanken nicht zur Seite schieben. Er leide stark unter diesen Fantasien und wisse nicht, wie er mit der zunehmenden Dranghaftigkeit umgehen könne. Er befürchte, auch noch seinen Job und sein zweites Studium $\mathrm{zu}$ verlieren. Aufgrund der starken Verzweiflung und der erlebten Hilflosigkeit kam es bislang zu einem stationären Aufenthalt in einer psychiatrischen Klinik, damals habe er Suizidgedanken gehabt. Über seine eigentliche Problematik habe er sich jedoch nicht öffnen können. Aus lauter Verzweiflung habe er auch schon einmal sein Handy zerstört, da es ihm zu viel geworden sei, mit den Missbrauchsabbildungen und der zwanghaften Masturbation. Kurz darauf habe er sich jedoch ein neues Telefon besorgt und die entsprechenden Dateien wieder heruntergeladen. Aus seiner Sicht schaffe er es jedoch unter der Zuhilfenahme von Missbrauchsabbildungen, keinen Realkontakt zu Jungen aufzunehmen.

\section{Fazit}

In diesem Fall lässt sich eine pädophile Störung ggf. in Kombination mit einer sexuell zwanghaften Verhaltensstörung im Sinne einer sog. schweren anderen seelischen Abartigkeit diskutieren. Die psychosoziale Leistungsfä- 
higkeit imponiert krankheitsbedingt deutlich beeinträchtigt. Der zunehmende und anhaltende Konsum von Missbrauchsabbildungen führte zu beruflichen Problemen und gefährdete nachvollziehbar das Vorankommen im Studium. In einem zweiten Schritt finden sich Anhaltspunkte dafür, dass Herr B. möglicherweise relevant vermindert steuerungsfähig war. Herr B. konnte aufkommenden sexuellen Impulsen wenig entgegensetzen, reagierte mit sofortiger Masturbation in Kombination von ständigem Gebrauch der Missbrauchsabbildungen, sogar in sozial kontrollierten Situationen (öffentliche Toilette). Damit erscheint eine sexuelle Dranghaftigkeit, wie oben beschrieben, vorzuliegen. Daneben finden sich Hinweise für einen emotionalen Zusammenbruch im Zusammenhang mit der Nutzung der Missbrauchsabbildungen und der Paraphilie (Krankenhausaufenthalt, Suizidgedanken, Handy zerstört). Die soziale Vereinsamung kann dem Merkmal Zusammenbruch sozialer Unterstützung zugeordnet werden. Das Eingeständnis des Herrn B., mit den Impulsen und Wünschen überfordert gewesen zu sein und diesen nichts entgegensetzen zu können, demnach diesen ausgeliefert zu sein, korrespondiert mit Einschränkungen in der motivationalen Steuerungsfähigkeit als auch mit einer Verminderung der Desaktualisierungspotenz. Die unmittelbare Befriedigung von sexuellen Wünschen prägt letztlich seinen Alltag, und es gelingt ihm nicht einmal, nach wiederholtem Eintritt negativer Konsequenzen seine Impulse zu hemmen. Diese Einschränkungen könnten daher zu einer Minderung der Steuerungsfähigkeit geführt haben. Die dauerhafte paraphile Okkupierung wird auch auf der Verhaltensebene beim ständigen Mitführen von Missbrauchsabbildungen deutlich.

Funding Open Access funding enabled and organized by Projekt DEAL.

Interessenkonflikt J. Fuß, A. Voulgaris und P. Briken geben an, dass kein Interessenkonflikt besteht.

Open Access Dieser Artikel wird unter der Creative Commons Namensnennung 4.0 International Lizenz veröffentlicht, welche die Nutzung, Vervielfältigung, Bearbeitung, Verbreitung und Wiedergabe in jeglichem Medium und Format erlaubt, sofern Sie den/die ursprünglichen Autor(en) und die Quelle ordnungsgemäß nennen, einen Link zur Creative Commons Lizenz beifügen und angeben, ob Änderungen vorgenommen wurden.

Die in diesem Artikel enthaltenen Bilder und sonstiges Drittmaterial unterliegen ebenfalls der genannten Creative Commons Lizenz, sofern sich aus der Abbildungslegende nichts anderes ergibt. Sofern das betreffende Material nicht unter der genannten Creative Commons Lizenz steht und die betreffende Handlung nicht nach gesetzlichen Vorschriften erlaubt ist, ist für die oben aufgeführten Weiterverwendungen des Materials die Einwilligung des jeweiligen Rechteinhabers einzuholen.

Weitere Details zur Lizenz entnehmen Sie bitte der Lizenzinformation auf http://creativecommons.org/licenses/by/4.0/deed.de.

\section{Literatur}

Boetticher A, Nedopil N, Bosinski HA, Saß H (2007) Mindestanforderungen für Schuldfähigkeitsgutachten. Forens Psychiatr Psychol Kriminol 1(1):3-9

Briken P (2020) An integrated model to assess and treat compulsive sexual behaviour disorder. Nat Rev Urol 17:391-406. https://doi. org/10.1038/s41585-020-0343-7

Briken P, Müller JL (2014) Beurteilung der Schuldfähigkeit bei paraphiler Störung. Nervenarzt 85(3):304-311

Brunner F, Müller JL, Vogel S, Briken P (2016) Evaluation von operationalisierten Kriterien zur Schuldfähigkeitsbeurteilung bei paraphiler Störung. Recht Psychiatr 34(4):228-236

Dekker A, Koops T, Briken P (2016) Sexualisierte Grenzverletzungen und Gewalt mittels digitaler Medien. Expertise zur Bedeutung digitaler Medien für Phänomene sexualisierter Grenzverletzungen und Gewalt gegen Kinder und Jugendliche. Unabhängiger Beauftragter für Fragen des sexuellen Kindesmissbrauchs, Berlin

Dobbrunz S, Brunner F, Müller JL, Briken P (2020) Interrater-Reliabilität der kriteriengeleiteten Beurteilung der Schuldfähigkeit bei paraphilen Störungen. Nervenarzt. https://doi.org/10.1007/ s00115-020-00920-1

Franke I, Graf M (2016) Kinderpornografie. Forens Psychiatr Psychol Kriminol 10(2):87-97

Fuss J, Lemay K, Stein DJ, Briken P, Jakob R, Reed GM, Kogan CS (2019) Public stakeholders' comments on ICD-11 chapters related to mental and sexual health. World Psychiatry 18(2):233

Giese H (1962) Psychopathologie der Sexualitat. Enke, Stuttgart

Graf M, Dittmann V (2009) Konsumenten illegaler Internet-Pornographie - psychische Auffälligkeiten und Risiken der Straffälligkeit. Forens Psychiatr Psychol Kriminol 3(2):99-106

Janzarik W (1988) Strukturdynamische Grundlagen der Psychiatrie. Enke Verlag, Stuttgart

Janzarik W (1993) Steuerung und Entscheidung, deviante Strukturierung und Selbstkorrumpierung im Vorfeld affektiv akzentuierter Delikte. In: Saß H (Hrsg) Affektdelikte. Springer, Berlin Heidelberg New York, S 57-76

Konrad N (2018) Zum Krankheitsbegriff in der Forensischen Psychiatrie. Rechtspsychologie 4(1):40-54

Kröber HL (1993) Personlichkeit, konstellative Faktoren und die Bereitschaft zum „Affektdelikt“. In: Saß H (Hrsg) Affektdelikte. Springer, Berlin Heidelberg New York, S 77-94

Kröber HL (2007) Steuerungsfähigkeit und Willensfreiheit aus psychiatrischer Sicht. Handbuch der Forensischen Psychiatrie, Bd. 1, S $159-219$

Kröber HL (2016) Die Beurteilung der Steuerungsfähigkeit bei psychischen Störungen. Forens Psychiatr Psychol Kriminol 10(3):181-188

Matthes A, Rettenberger M (2008) Die deutsche Version des ACUTE2007 zur dynamischen Kriminalprognose bei Sexualstraftatern. Institut für Gewaltforschung und Pravention, Wien

$\mathrm{Saß}$ H (1991) Forensische Erheblichkeit seelischer Störungen im psychopathologischen Referenzsystem. In: Medizinrecht - Psychopathologie - Rechtsmedizin. Springer, Berlin, Heidelberg, S 266-281

Schorsch E (1988) Affekttaten und sexuelle Perversionstaten im strukturellen und psychodynamischen Vergleich. In: Der Mensch in der Psychiatrie. Springer, Berlin, Heidelberg, S 344-358

Taylor M, Holland G, Quayle E (2001) Typology of paedophile picture collections. Police J 74:97 